\title{
Direct observation of tetrahertz electromagnetic waves emitted from intrinsic Josephson junctions in single crystalline $\mathrm{Bi}_{2} \mathrm{Sr}_{2} \mathrm{CaCu}_{2} \mathrm{O}_{8+\delta}$
}

\author{
K. Kadowaki ${ }^{\text {a,b,* }}$, H. Yamaguchi ${ }^{\text {a,b }}$, K. Kawamata ${ }^{\text {a,b }}$, T. Yamamoto ${ }^{\text {a,b }}$, H. Minami ${ }^{\text {a,b }}$, \\ I. Kakeya ${ }^{\mathrm{a}, \mathrm{b}}$, U. Welp ${ }^{\mathrm{c}}$, L. Ozyuzer ${ }^{\mathrm{c}, \mathrm{d}}$, A. Koshelev ${ }^{\mathrm{c}}$, \\ C. Kurter ${ }^{\mathrm{c}, \mathrm{e}}$, K.E. Gray ${ }^{\mathrm{c}}$, W.-K. Kwok ${ }^{\mathrm{c}}$ \\ ${ }^{a}$ Institute of Materials Science, and Graduate School of Pure and Applied Sciences, University of Tsukuba, 1-1-1, Tennodai, Tskuba Ibaraki 305-8573, Japan \\ ${ }^{\mathrm{b}}$ CREST, JST, Japan \\ ${ }^{\mathrm{c}}$ Materials Science Division, Argonne National Laboratory, Argonne, IL 60439, USA \\ ${ }^{\mathrm{d}}$ Department of Physics, Izmir Institute of Technology, TR-35430 Izmir, Turkey \\ ${ }^{\mathrm{e}}$ Disision, Illinois Institute of Technology, Chicago, IL 60616, USA
}

Accepted 30 November 2007

Available online 13 March 2008

\begin{abstract}
We have observed intense, coherent, continuous and monochromatic electromagnetic (EM) emission at terahertz frequencies generated from a single crystalline mesa structure of the high- $T_{\mathrm{c}}$ superconductor $\mathrm{Bi}_{2} \mathrm{Sr}_{2} \mathrm{CaCu}_{2} \mathrm{O}_{8+\delta}$ intrinsic Josephson junction system. The mesa is fabricated by the Argon-ion-milling and photolithography techniques on the cleaved surface of $\mathrm{Bi}_{2} \mathrm{Sr}_{2} \mathrm{CaCu}_{2} \mathrm{O}_{8+\delta}$ single crystal. The frequency, $v$, of the EM radiation observed from the sample obeys simple relations: $v=c / n \lambda=c / 2 n w$ and $v=2 e V / h N$, where $c$ is the light velocity in vacuum, $n$ the refractive index of a superconductor, $\lambda$ the wave length of the EM emission in vacuum, $w$ the shorter width of the mesa, $V$ the voltage applied to the mesa, $N$ the number of layers of intrinsic Josephson junctions, $e$ and $h$ are the elementary charge and the Planck constant, respectively. These two relations strongly imply that the mechanism of the emission is, firstly, due to the geometrical resonance of EM waves to the mesa like a cavity resonance occuring in the mesa structure, and forming standing waves as cavity resonance modes, and secondly, due to the ac-Josephson effect, which works coherently in all intrinsic Josephson junctions. The peculiar temperature dependence of the power intensity emitted form samples shows a broad maximum in a temperature region between 20 and $40 \mathrm{~K}$, suggesting that the nonequilibrium effect plays an essential role for the emission of EM waves in this system. The estimated total power is significantly improved in comparison with the previous report [L. Ozyuzer et al., Science 318 (2007) 1291, K. Kadowaki, et al., Physica C 437-438 (2006) 111, I.E. Batov, et al., Appl. Phys. Lett. 88 (2006) 262504], and reached as high as $5 \mu$ W from single mesa with $w=60 \mu \mathrm{m}$ at $648 \mathrm{GHz}$, which enables us to use it for some of applications. So far, we succeeded in fabricating the mesa emitting EM waves up to $960 \mathrm{GHz}$ in the fundamental mode in the $w=40 \mu \mathrm{m}$ mesa, whereas the higher harmonics up to the 4-th order were observed, resulting in a frequency exceeding $2.5 \mathrm{THz}$. In sharp contrast to the previous reports [K. Kadowaki, et al., Physica C 437-438 (2006) 111 , M.-H. Bae, et al., Phys. Rev. Lett. 98, (2007) 027002], all the present measurements were done in zero magnetic field. Lastly, a plausible theoretical model for the mechanism of emission is discussed.
\end{abstract}

(C) 2008 Elsevier B.V. All rights reserved.

PACS: $07.57 . \mathrm{Hm} ;$ 74.50.+r; 85.25.Cp

Keywords: THz emission; Intrinsic Josephson junction; ac-Josephson effect; Harmonics

\footnotetext{
* Corresponding author. Address: Institute of Materials Science, and Graduate School of Pure and Applied Sciences, University of Tsukuba, 11-1, Tennodai, Tskuba Ibaraki 305-8573, Japan. Tel.: +81 (0) 29853 5291; fax: +81 (0) 298534490 .

E-mail address: kadowaki@ims.tsukuba.ac.jp (K. Kadowaki).
}

\section{Introduction}

Although electromagnetic waves have been used in a vast of applications and have been considered as the most 
developed scientific and technological fields covering over the wide frequency range from radio frequencies to visible light, X-ray and gamma-ray regions in the electromagnetic spectrum, a narrow window region at terahertz frequencies is surprisingly missing and remains underdeveloped even at present day, leaving a stigmatic word as "THz technology gap" [6,7]. The reason for this is apparent and simply stems from the lack of good oscillator sources as well as the sensitive detectors in this frequency range despite of huge scientific and technological interest in applications [8].

Recently, we have succeeded in generating intense, coherent, continuous and monochromatic electromagnetic (EM) waves in the $\mathrm{THz}$ frequency region emitted from the mesa structure fabricated on the single crystalline high- $T_{c}$ superconductor $\mathrm{Bi}_{2} \mathrm{Sr}_{2} \mathrm{CaCu}_{2} \mathrm{O}_{8+\delta}$, which is known as a typical intrinsic Josephson junction system $[1,2]$. Here, we provide further detailed information on such fascinating phenomenon generating EM waves at $\mathrm{THz}$ frequencies mainly from experimental point of view in order to make it clear the mechanism of the emission. Since this observed phenomenon is entirely new and is very promising for the vast area of applications, development of this method may contribute to a key technology in the field of applications.

An interesting characteristic feature of this EM emission lies in a monochromatic spectrum $[1,2]$. The line width is so sharp that the observed line width of radiation is limited by the resolution of our conventional spectrometers which has $0.25 \mathrm{~cm}^{-1}$. Our sample was found to emit continuously in a period of time scale of a few hours at least with keeping a very stable power level, although the life time has not been tested yet. Furthermore, the frequency can be varied considerably, approximately $10-15 \%$ of the center frequency. This variable range of frequency, $\Delta v$, strongly differs from sample to sample, depending on the preparation conditions. Although we are not able to control of this range, $\Delta v$, at this stage of study, this experimental fact certainly means that the emission frequency can be tunable within approximately $10-15 \%$ of the central frequency.

The central frequency can be adjusted by the size of the width, $w$, of the rectangular mesa sample. We so far have fabricated several mesa samples successfully, which have the emission frequencies of $v=357,480,560,648$ and $870 \mathrm{GHz}$ for the mesas with $w=100,80,60,60$ and $40 \mu \mathrm{m}$, respectively. This frequency obeys nicely the following relation:

$v=c / n \lambda=c / 2 n w$

and

$v=2 \mathrm{eV} / \mathrm{hN}$,

where $c$ is the light velocity in vacuum, $n$ the refractive index of superconductor, $\lambda$ the wave length of the EM emission in vacuum, $w$ the shorter width of the mesa, $V$ the voltage applied to the mesa, $N$ the number of layers of intrinsic Josephson junctions, $e$ and $h$ are the elementary charge and the Planck constant, respectively. This relation strongly implies that the mechanism of the emission is, firstly, due to the geometrical resonance of EM waves to the mesa, which seems to work as a cavity, and to form standing waves in it as the cavity resonance modes. Secondly, the $a c$-Josephson effect works as a essential mechanism of generation of the coherent EM waves from intrinsic Josephson junctions.

In addition to the central line given by the conditions of Eqs. (1) and (2), higher order harmonics associated with the central line are clearly observed even up to the 4-th order [2]. For example, based on the fundamental mode of $v=0.64 \mathrm{THz}$, the 4-th harmonics results in $2.56 \mathrm{THz}$, though the intensities of higher harmonics become weaker and weaker. Since the mechanism of appearance of the higher harmonics strongly depends on the mechanism of the central emission line, it is expected that the understanding of the higher harmonics may lead us to identify the mechanism of emission of $\mathrm{THz}$ waves in this system [2,9]. Therefore, we show experimental results on the higher harmonics obtained recently in the mesa sample with $60 \mu \mathrm{m}$ width in detail.

Finally, we estimate the total power emitted from the mesa sample by careful consideration of experimental setup as well as the environmental absorption effect, etc. As will be discussed, the emission power is temperature dependent, having a peak mostly between 30 and $45 \mathrm{~K}$, depending on the sample. Fixing temperature at which the power is strongest, the total power is estimated to be as strong as about $5 \mu \mathrm{W}[2,9]$. This level of power is already sufficient for some useful applications.

\section{Samples}

The samples were prepared from a piece of single crystal grown by the traveling floating zone method [10]. A cleaved thin single crystal with a thickness of several $\mu \mathrm{m}$ was glued on a sapphire substrate by U-vanish (polymide resin), and a silver thin layer and a gold film are sputtered on the cleaved sample surface. Then, the sample mesa and two contact pads with a desired size were patterned by the photolithography and Argon ion milling techniques. After removing unnecessary resist, $\mathrm{CaF}_{2}$ is evaporated on the part of mesa for the electrical isolation purpose, and $\mathrm{Au}$ is finally deposited for the contact pad. This Au is again patterned by photolithography and Argon milling techniques again. The detailed process for the sample preparation will be described separately.

The SEM photographs of the sample is shown in Fig. 1. as an example. It is noted that the sample is not rectangular but a considerable trapezoidal shape after Argon milling.

The $\mathrm{Au}$ wire with $10 \mu \mathrm{m}$ in diameter for electrical connection was attached to the mesa by $\mathrm{Ag}$ paste as shown in Fig. 1B, and also for the other two contact pads.

All measurements such as $I-V$ curves, temperature dependence of resistance, etc. have been measured with the three terminal method. 

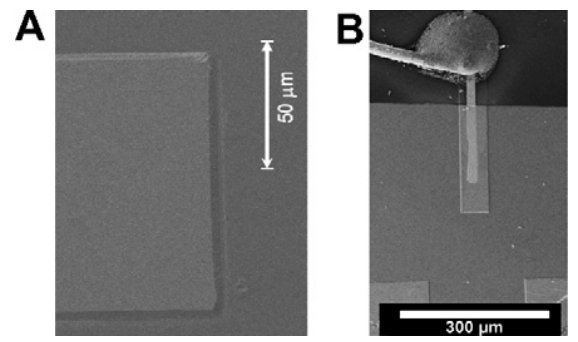

Fig. 1. (A) A photograph of $w=100 \mu \mathrm{m}$ mesa with $300 \mu \mathrm{m}$ length. (B) A photograph of $w=60 \mu \mathrm{m}$ mesa with $300 \mu \mathrm{m}$ length with electrical contacts. One electrical lead taken from the gold contact pad at the top of the mesa and two leads (not visible) are taken from the gold contact pads at the bottom of the mesa. The heights of the terrace in both cases are $1 \mu \mathrm{m}$.

\section{Experimental set-up}

The $I-V$ curves have been measured with the conventional set-up for resistance measurements. The block diagram of the circuit used in the present measurement was shown in Fig. 2. A multipurpose function generator was used for generating ramping current for the sample. For measurement of the full $I-V$ curve, this enables us to supply a maximum voltage with a few volts to the sample at a ramp rate from $0.1 \mathrm{mHz}$ to $100 \mathrm{~Hz}$. The current was monitored by the voltage drop through $10 \Omega$ standard resistor by a commercial digital multimeter (DMM1). The output voltage from the sample was measured by the DMM2, and both are simultaneously displayed on the oscilloscope. The output data of DMM1 and DMM2 were stored in the computer (PC) through the IEEE 488 bus. The bias condition for the mesa sample was adjusted by the resistor $R=10-150 \Omega$.

For the detection and spectroscopy of the EM emission a Si-bolometer cooled at $4.2 \mathrm{~K}$ and two FT-IR spectrometers (Bruker ISF66V and JASCO FARIS-1) with Si-bolometer were used. The block diagram is shown in Fig. 3. Firstly, the emission from the sample was checked using circuit block $\mathrm{A}$ and $\mathrm{B}$ while the $I-V$ curve was measured with a very slow current scan $(0.1-1 \mathrm{mHz})$. When the emission is confirmed by the Si-bolometer A, the ramp of the current is stopped and the shutter is closed, then the FTIR measurement is performed using the circuit block C.

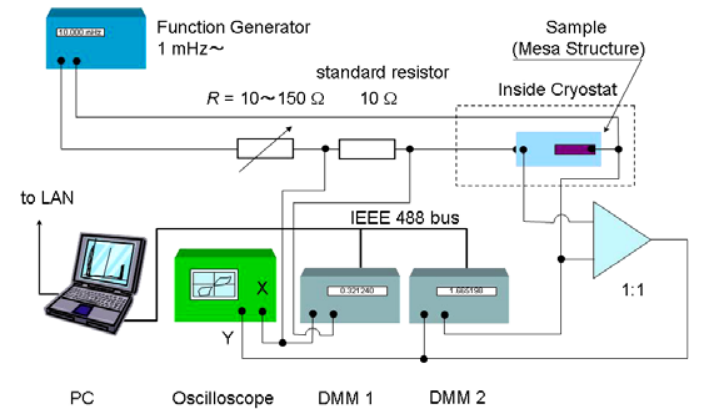

Fig. 2. The block diagram of the electrical measurements.

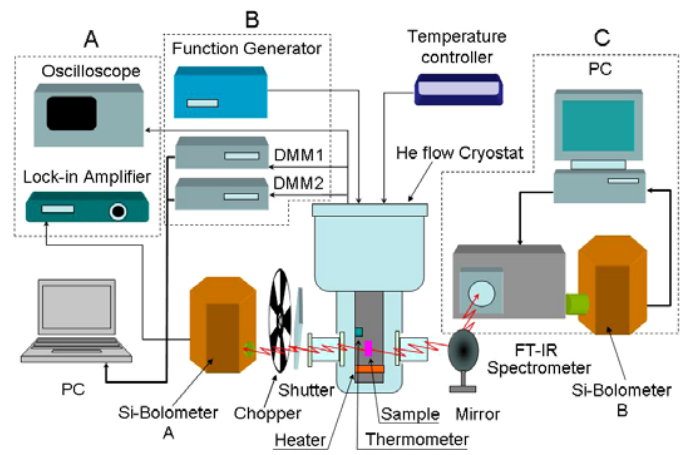

Fig. 3. The block diagram of the emission detection system. Two $\mathrm{Si}$ bolometers A and B were used. The components A are used for the detection of emission from the sample. The chopper frequency was set in between 70 and $80 \mathrm{~Hz}$. The components $\mathrm{B}$ are used for the electrical measurements such as $I-V$ curves. The components $\mathrm{C}$ are used for the FTIR spectroscopic purposes. The sample is cooled by the liquid He flow cryostat.

If necessary, a half sphere $\mathrm{Si}$ lens is inserted just in front of the sample in order to focus the beam. It is noted that for detection of the $\mathrm{THz}$ emission, it is important to avoid air in the optical path because of strong absorption due mainly to the water vapor. Therefore, we enclosed the whole set-up including the EM emission paths by the plastic bag which is filled by pure nitrogen gas.

\section{Experimental results}

\subsection{Temperature dependence of the mesa resistance}

Before going into the detailed study, the temperature dependence of the mesa resistance is always measured. This gives very useful information to check the condition of the mesa such as the contact resistance, the sample quality, the doping level, etc. In Fig. 4, two examples are shown. The curves exhibit a typical temperature dependence of the $c$-axis resistance of $\mathrm{Bi}_{2} \mathrm{Sr}_{2} \mathrm{CaCu}_{2} \mathrm{O}_{8+\delta}$, which tends to increase with decreasing temperature around room temperature and turns to sharp increase near $T_{\mathrm{c} \text {. }}$ Our samples so

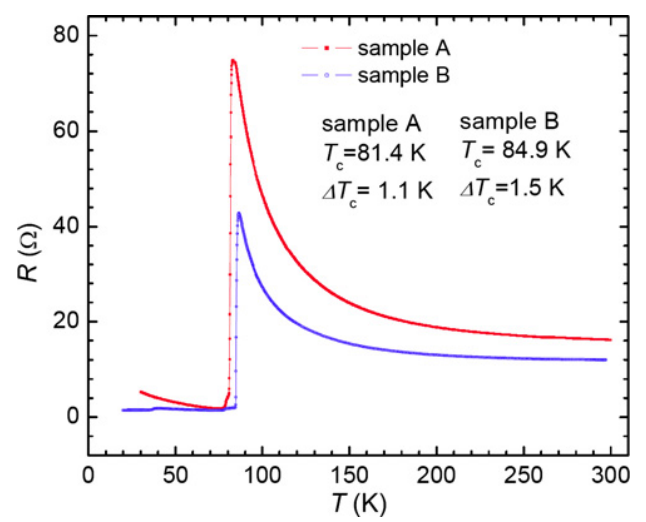

Fig. 4. The temperature dependence of the resistance of two typical $\mathrm{Bi}_{2} \mathrm{Sr}_{2} \mathrm{CaCu}_{2} \mathrm{O}_{8+\delta}$ mesas. The sample $\mathrm{A}$ is more underdoped than the sample $\mathrm{B}$ (qualitatively). 
far prepared have $T_{\mathrm{c}}$ between 75 and $85 \mathrm{~K}$, which indicate that the mesa is in the under-doped condition. The transition width, $\Delta T_{\mathrm{c}}$, is sharp and is mostly $1-1.5 \mathrm{~K}$.

As is seen in Fig. 4 the mesa resistance is finite even below $T_{\mathrm{c}}$. This is because of the contact surface resistance due to three terminal measurement. However, the contact resistance is usually smaller than a few Ohms so that it seems not to influence the measurement, but it may contribute to the heating, which will be discussed below.

\subsection{The $I-V$ curves}

The current-voltage $(I-V)$ relations were measured in all samples at the beginning of the experiment. They show a number of characteristic features of the multistacked intrinsic Josephson junction system and some unusual features, perhaps, associated with the mesa junctions used in particular in this experiment. Firstly, the size of the mesa with a few $100 \mu \mathrm{m}$ by several tens of $\mu \mathrm{m}$ is considerably larger in size than the ones used in all previous works.

An example of the $I-V$ curves are shown in Fig. 5, where 5 curves are overlaid with slightly different maximum currents applied to the $w=60 \mu \mathrm{m}$ mesa measured at $29 \mathrm{~K}$. At smaller currents and voltages the curves jump stepwise many times until they reach a big jump, which corresponds to the quasiparticle state of whole intrinsic junctions. When the current is returned before reaching the big jump, the $I-$ $V$ curves are smoothly come back to zero voltage, resulting in a big hysteresis loop, as seen in Fig. 5. It is noted that the stepwise increasing behavior of the voltage of the junction with increasing current does not always trace the same path but it jumps rather randomly. On the contrary to this it is rather smooth on the way of the return branch.

When the current exceeds a certain threshold value, a catastrophic change happens: the $I-V$ curve shows a big jump to the full quasiparticle state. Once this catastrophy happens the junction resistance show a very strong negative resistance behavior and significant temperature increase of the junction is observed. This is due to heating by the quasiparticle current flowing through a couple of hundreds

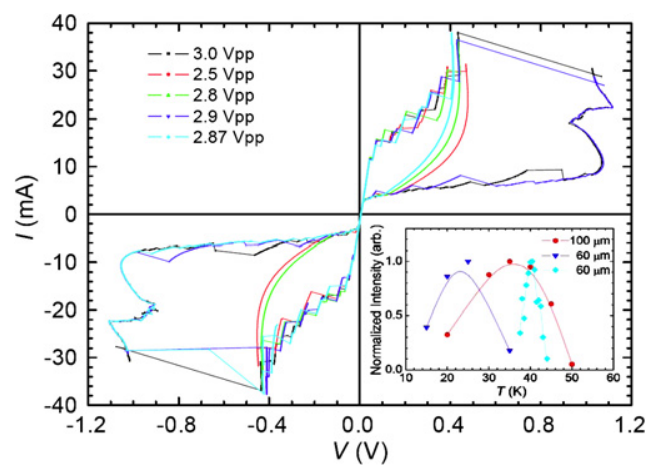

Fig. 5. The five $I-V$ curves with different maximum currents (corresponding maximum voltage are shown) are overlaid. In the inset of right bottom corner the normalized power intensity is plotted as a function of temperature for three typical samples with different size of $w=60,80$ and $100 \mu \mathrm{m}$.
Ohm junction resistance, and it is estimated to generate heat of a few tens of $\mathrm{mW}$ or even more, depending on the junction resistance and the thermal conductivity through the contact surfaces of the samples, the substrate and the copper sample holder, etc. This heat is huge so that the temperature of the sample easily rises much higher than that of the sample holder. Most of the cases above the catastrophic jump the junction temperature is elevated even above $T_{\mathrm{c}}$.

When the current is reduced from the catastrophic state gradually, the $I-V$ curve follows a large hysteretic negative resistance curve, then finally comes back to the positive resistive slope. In the middle of the curve where the resistance changes the slope from negative to positive and a strong anomaly occurs with a sharp voltage dip, strong EM emission is observed. Note that this sort of sharp dip in voltage does not always happen. The EM emission can also be observed even without such a sharp voltage dip in some samples. So far, it is not clear the reason of this sharp voltage dip, whether it is related to the mechanism of EM emission or not. Further detailed study is needed to understand this phenomenon.

The whole $I-V$ curve is displayed again in Fig. 6A, while a sharp dip region in voltage with strong EM emission indicated by a circle in Fig. 6A, is replotted in Fig. 6B in an expanded scale together with the detected radiation power. It is interesting to note that the emission occurs at the both decreasing and increasing voltages in the sharp dip region with the noticeable current-voltage jittering fluctuations just before the occurrence of emission, while the current is continuously decreasing. Once the EM radiation begins the emission continues steadily, at least over $2 \mathrm{~h}$. Moreover, the power level is surprisingly constant within $3.6 \times 10^{-3}$.

\subsection{Temperature dependence}

One peculiar phenomenon observed in all samples so far measured is that the EM emission only occurs at a limited temperature region, which lies between 15 and $50 \mathrm{~K}$. Some samples have a rather sharp and an only $10 \mathrm{~K}$ width or so. This peculiar behavior is shown in the inset of Fig. 5, for
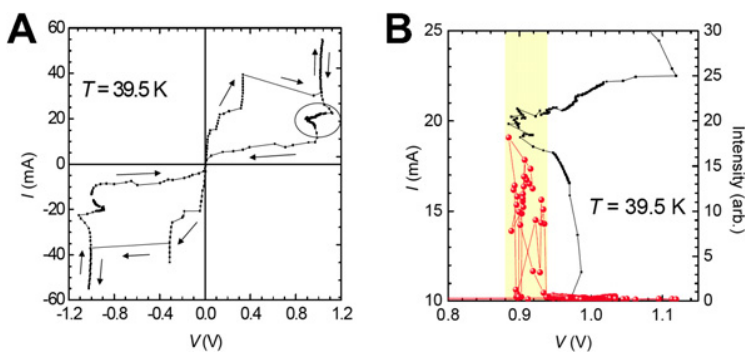

Fig. 6. (A) The $I-V$ curve taken at $39.5 \mathrm{~K}$, where the emission is maximum. A circle indicates the region with a sharp voltage dip where strong emission occurs. (B) The region encircled in Fig. 6A is shown in an expanded scale. The emitted power intensity is also added as a function of the applied voltage. 
three typical samples. Although we do not understand the reason clearly, it is speculated that such a strong temperature dependence may originate from the nonequilibrium effect due to the inhomogeneous temperature distributions occuring in the junction. In addition, such nonequilibrium condition may be favorable for and may actually trigger the nonlinear resonant oscillations of the supercurrent flow. It is reminded that the temperature of the junction itself is much higher than the bath temperature indicated in Fig. 5.

\subsection{Frequency spectrum}

The frequency spectrum were measured by two FTIR spectrometers with different beam splitters; one is the Bruker ISF66V with mylar beam splitter (Michelson type interferometer), which can go down to $20 \mathrm{~cm}^{-1}$, while the other is the JASCO FARIS-1 with a wire grid beam splitter (Martin-Puplett type interferometer), which can measure down to $2 \mathrm{~cm}^{-1}$, respectively. The resolution of both spectrometers has the same as $0.25 \mathrm{~cm}^{-1}$.

When emission is detected at a certain current and voltage level, then the spectometer is operated at the fixed current by the multiple scan mode. The signal is averaged by 16-62 times, depending on the condition.

Fig. 7 shows a typical result of the spectrum of the emission observed in $w=60 \mu \mathrm{m}$ mesa at $40 \mathrm{~K}$. Here, we find several interesting features associated with the spectrum. First of all, as seen in Fig. 7 the line width of the emission is very sharp. It seems to be less than the instrumental resolution limit, which is nominally $\Delta k=0.25 \mathrm{~cm}^{-1}=$ $7.5 \mathrm{GHz}$ in both spectrometers. To show this more clearly, the spectrum obtained in the case of different sample with $w=100 \mu \mathrm{m}$ sample is displayed in an expanded scale in the inset of Fig. 7. Here, the full width at half maximum of the emission line is measured to be $0.374 \mathrm{~cm}^{-1}$, which corresponds to the $11.2 \mathrm{GHz}$ in frequency. Although this value is somewhat larger than the instrumental resolution of $0.25 \mathrm{~cm}^{-1}$, this is attributed to the instrumental origin.

Secondly, as seen in Fig. 7, the fundamental emission is found to accompany the higher harmonics up to the 4-th

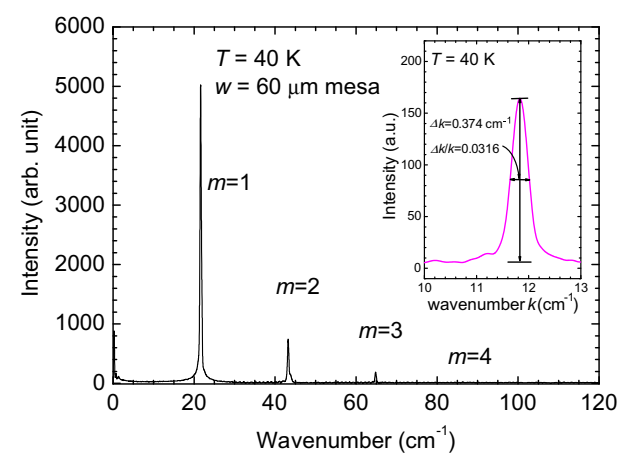

Fig. 7. The spectrum of the emission observed from the $w=60 \mu \mathrm{m}$ mesa made from $\mathrm{Bi}_{2} \mathrm{Sr}_{2} \mathrm{CaCu}_{2} \mathrm{O}_{8+\delta}$ at $40 \mathrm{~K}$. The intense fundamental mode with the frequency expected form Eqs. (1) and (2) accompanied by the higher harmonics up to the 4 th order can clearly be seen.
Table 1

The characteristics of the higher harmonics; wavenumbers and the corresponding widths, frequencies and the corresponding widths, intensities of the spectrum shown in Fig. 7 are listed

\begin{tabular}{llll}
\hline$m$ & $K\left(\mathrm{~cm}^{-2}\right)$ & $v(\mathrm{THz})$ & $I(\mathrm{arb})$. \\
& $\Delta k\left(\mathrm{~cm}^{-2}\right)$ & $\Delta v(\mathrm{GHz})$ & \\
\hline$m=1$ & $21.623 \pm 0.005$ & $0.64825 \pm 0.0002$ & 4899 \\
& $0.371 \pm 0.004$ & $11.2 \pm 0.01$ & \\
$m=2$ & $43.2311 \pm 0.005$ & $1.2960 \pm 0.0002$ & 385 \\
& $0.399 \pm 0.004$ & $12.0 \pm 0.01$ & \\
$m=3$ & $64.828 \pm 0.005$ & $1.94351 \pm 0.0002$ & 101 \\
& $0.396 \pm 0.004$ & $11.9 \pm 0.01$ & \\
$m=4$ & $86.36 \pm 0.02$ & $2.5891 \pm 0.0006$ & $18 \pm 5$ \\
& 0.31 & 9.4 & \\
\hline
\end{tabular}

order in this particular case. The intensity of the higher harmonics seems to decay exponentially, whereas the line width remains unchanged; as sharp as the fundamental one. The frequencies of the fundamental one as well as the higher harmonics determined experimentally are listed in the Table 1. As seen from the Table 1 the ratios of the frequencies of the higher harmonics to the fundamental one are $v_{2} / v_{1}=1.9993, v_{3} / v_{1}=2.9981$ and $v_{4} / v_{1}=3.9940$, while the intensity ratios are $I_{2} / I_{1}=0.78, I_{3} / I_{1}=0.021$ and $I_{4} / I_{1}=0.0037$. These numbers of the frequency ratios of higher harmonics to the fundamental one are extraordinary close to the integers within the instrumental error. It is noted that the emission frequency does not depend on temperature to the noticeable level, although the temperature range is restricted to the narrow region.

\subsection{Estimation of emission power}

Finally, we estimate the total power, $P$, emitted form the sample. The EM waves emitted from the sample propagate through the window of the cryostat made of polyethylene, the chopper with $50 \%$ transmission efficiency, the window of the bolometer with the diameter of $1.27 \mathrm{~cm}$ made of polyethylene, the low pass filter of $300 \mathrm{~cm}^{-1}$ and reach the Si-bolometer. The power at the Si detector, $P_{\text {obs }}$, can be written as $P=\varepsilon_{\mathrm{eff}} P_{\mathrm{obs}}$, where $\varepsilon_{\mathrm{eff}}$ is the efficiency coefficient. This value can be estimated to be $\varepsilon_{\mathrm{eff}}=5.7 \times 10^{3}$ only from the geometrical configuration of the equipments. The observed power, $P_{\text {obs }}$, can be written as $P_{\text {obs }}=V_{\text {out }} / \mathrm{S}$, where $V_{\text {out }} \approx 0.2 \mathrm{mV}$ is the output voltage from the $\mathrm{Si}$ bolometer on the maximum emission of the sample, and $S=2.19 \times 10^{5} \mathrm{~V} / \mathrm{W}$ is the calibration constant of the Sibolometer. This, therefore, leads to a value of $P \sim 5 \mu \mathrm{W}$. Since the total power fed into the mesa is approximately $15 \mathrm{~mW}$ in this case, this results in an total efficiency of radiation to be $\sim 3 \times 10^{-4}$. However, to be more precise, the angular dependence of the emission power should be taken into account carefully [9].

\section{Conclusions}

In conclusion, we have presented a novel approach to generate intense, continuous, coherent and monochromatic 
EM waves at $\mathrm{THz}$ frequencies by making use of mesas fabricated from intrinsic Josephson junctions of single crystalline $\mathrm{Bi}_{2} \mathrm{Sr}_{2} \mathrm{CaCu}_{2} \mathrm{O}_{8+\delta}$ by a combination of the ion milling and photolithography techniques. We succeeded in observation of the intense power of EM emission of $\sim 5 \mu \mathrm{W}$, which have never been achieved before. It is indeed 5-6 orders of magnitude stronger than that from single Josephson junction, and even a couple of orders of magnitude stronger than the previous reports [3-5]. For the emission it is required to fulfill two conditions: Eqs. (1) and (2) at the same time. The higher harmonics were observed up to $2.5 \mathrm{THz}$ in the 4-th harmonics in the case of the $w=60 \mu \mathrm{m}$ mesa sample. Since the emission mechanism of $\mathrm{THz}$ waves is not completely established yet, it is certainly important to experimentally study the angular dependence of the higher harmonics and their intensity relations, which may give us a important clue to identify the mechanism. It is, perhaps, interesting to mention one theoretical model based on the Josephson nonlinearity. In this model the total Josephson current, $J=J_{\mathrm{c}} \sin \theta(t)=$ $J_{\mathrm{c}} \sin \left(\omega_{j} t+v \sin \omega_{j} t\right)$, which can be expanded by linear combinations of the harmonics, $\left\{J_{\mathrm{n}-1}(v) \pm J_{\mathrm{n}+1}(v)\right\} \sin n \omega_{\mathrm{J}} t$, where $J_{\mathrm{c}}$ is the critical current of the junction, $v$ the ratio of the $a c$ Josephson voltage to the static voltage $V, \omega_{J}$ the $a c$ Josephson frequency, $J_{\mathrm{n}}(v)$ the $n$-th order of Bessel function. Since the total emission power, $P$, is proportional to the square of the spatial average of the total $J$, the intensity of the higher harmonics strongly depend on the spatial variation of $v$, which determines the resonance mode of the $\mathrm{THz}$ waves in the mesa sample. Therefore, it is crucial to analyze the higher harmonics in detail to resolve the mechanism of the EM emission in this system [11-13].

\section{Acknowledgements}

We deeply indebt to Prof. M. Tachiki, Dr. X. Hu, Dr. A. Yurgens, Dr. P. Warburton, Dr. G.W. Crabtree, Dr. A. Kanda and Prof. Ootuka and thank them with stimulating discussion. This work has been supported in part by JST (Japan Science and Technology Agency) CREST project, also in part by the JSPS (Japan Society for the Promotion of Science) CTC program and by the Grant-in-Aid for Scientific Research (A) under the Ministry of Education, Culture, Sports, Science and Technology (MEXT) of Japan. Work at Argonne was supported by the US-DoE under contract No. DE-AC02-06CH11357.

\section{References}

[1] L. Ozyuzer et al., Science 318 (2007) 1291.

[2] K. Kadowaki, et al., Phys. Rev. Lett., submitted for publication.

[3] I.E. Batov et al., Appl. Phys. Lett. 88 (2006) 262504.

[4] K. Kadowaki et al., Physica C437-438 (2006) 111.

[5] M.-H. Bae et al., Phys. Rev. Lett. 98 (2007) 027002.

[6] R. Kleiner, Science 318 (2007) 1254.

[7] M. Tonouchi, Nat. Photon. 1 (2007) 97.

[8] For instance G. Gruner (Ed.), Millimeter and Submillimeter Wave Spectroscopy of Solids, Springer Verlag, 1998.

[9] H. Minami, et al., in preparation.

[10] T. Mochiku, K. Kadowaki, Physica C 235-240 (1994) 523.

[11] M. Tachiki, et al., in preparation.

[12] A.E. Koshelev, L.N. Bulaevskii, cond-mat/07083269.

[13] A.E. Koshelev, in preparation. 